\title{
An Image Detection Method Based on Parameter Optimization of Support Vector Machine
}

\author{
Zhanshen Feng \\ Department of Information Engineering, Xuchang University, Xuchang 461000, Henan, China \\ Received: August 17, 2020. Revised: March 20, 2021. Accepted: April 6, 2021. Published: April 8, 2021.
}

\begin{abstract}
With the progress and development of multimedia image processing technology, and the rapid growth of image data, how to efficiently extract the interesting and valuable information from the huge image data, and effectively filter out the redundant data, these have become an urgent problem in the field of image processing and computer vision. In recent years, as one of the important branches of computer vision, image detection can assist and improve a series of visual processing tasks. It has been widely used in many fields, such as scene classification, visual tracking, object redirection, semantic segmentation and so on. Intelligent algorithms have strong non-linear mapping capability, data processing capacity and generalization ability. Support vector machine (SVM) by using the structural risk minimization principle constructs the optimal classification hyper-plane in the attribute space to make the classifier get the global optimum and has the expected risk meet a certain upper bound at a certain probability in the entire sample space. This paper combines SVM and artificial fish swarm algorithm (AFSA) for parameter optimization, builds AFSA-SVM classification model to achieve the intelligent identification of image features, and provides reliable technological means to accelerate sensing technology. The experiment result proves that AFSA-SVM has better classification accuracy and indicates that the algorithm of this paper can effectively realize the intelligent identification of image features.
\end{abstract}

Keywords - Image Detection, Support Vector Machine, Artificial Fish Swarm Algorithm, Parameter Optimization.

\section{INTRODUCTION}

$\mathrm{I}^{\mathrm{n}}$ today's rapid development of intelligent information age, multimedia data information explosion growth, these data content involves a huge amount of image data, the rapid increase of these data has brought great challenges to the current image processing technology. Therefore, in the context of large-scale image data, it is necessary to actively improve the extraction efficiency of effective information of the image in order to carry out subsequent operations. Image detection is the process of extracting the interested feature area (detection target) through the image, in which the image is the carrier of the detection target, the detection target needs to be extracted and summarized in advance, and finally separated by the corresponding algorithm [1]. Inspired by the cognitive mechanism of the brain, image detection is an important part of computer vision. Its purpose is to objectively detect the most significant areas in the image and express the detected results in the form of binary graph. People have an outstanding ability to screen image information. They can quickly and selectively select some image information that they are interested in, while ignoring other visible information, they can concentrate limited perceptual and cognitive resources on the most relevant perceptual data subset [2]. In a word, image detection has a strong application value in today's social background, but it is also full of a variety of challenges, which is one of the problems that need to be solved effectively in the field of computer vision. Image detection methods can be divided into single frame image detection and multi frame image detection. Single frame image detection mainly uses the gray information of the image to segment the target, including the target detection method based on gray threshold and the target detection method based on edge information. Multi frame image detection is mainly used for moving object detection, including pixel analysis method, feature detection method and transformation based method. Support Vector Machines (SVM) is a binary classification model. Its basic model is the linear classifier with the largest interval defined in the feature space. The maximum interval makes it different from the perceptron; SVM also includes kernel techniques, which makes it a non-linear classifier in essence. The learning strategy of SVM is to maximize the interval, which can be formalized as a convex quadratic programming problem, which is equivalent to the minimization of the regularized hinge loss function. The learning algorithm of SVM is the optimization algorithm to solve convex quadratic programming. SVM is a quadratic programming problem of several basic equality constraints within a certain scope. So it has introduced the concept of slack variable, controlled the generalization ability of the model to stabilize it within a certain scope and effectively solve practical engineering problems through continuous trainings, optimization and iterations [3]. Artificial Fish Swarm Algorithm (AFSA) is a new-type intelligent optimization algorithm, which is a top-down optimization algorithm. The algorithm is based on the behavior of fish foraging, gathering and tailing, it has many strengths: it has strong robustness, powerful global search ability and the setting of parameter tolerance and it is also proven to be insensitive to the initial value. This paper has adopted AFSA in the parameter 
optimization of SVM and realized the image classification and intelligent feature identification.

At present, image detection technology is developed towards the rapid and accurate direction. In linearly separable circumstances, SVM searches the optimal classification hyper-plane of two types of samples in the original space. In linearly inseparable cases, SVM adds slack variable for analysis, maps the samples in low-dimensional input space into the high-dimensional attribute space through non-linear mapping to make it possible to adopt linear algorithms and analyze non-linearity of samples in high-dimensional attribute space, and searches the optimal classification hyper-plane in this feature space [4] [5]. In 1995, Vapnik had proposed SVM as the new pattern recognition method based on the statistical learning theory. Its main idea was to build a classification decision plane and SVM mapped the data into high-dimensional space via kernel function in order to make it as linearly separable as possible. In the same year, Vapnik and Cortes had come up with soft margin SVM, which increased a component in the objective function to punish non-zero slack variable by introducing the false classification of measurement data of slack variable [6]. In 1998, Weston and others had brought forward the Multi-Class Support Vector Machine (Multi-SVM). It has converted multi-class classification into binary classification and applied SVM into the judgment of multi-class problems. Suykens and others with the double helix classification problem of DNA analysis as the research object, proposed to simplify and converted inequality constraint problems into equality constraint problems based on the original SVM model and converted the especially complex SVM training process into the solution to the system of linear equations which has greatly reduced the time and difficulty it takes to build SVM [7]. Suykens had proposed the Least Square Support Vector Machine (LS-SVM) algorithm and Scholkoph and Smola had raised V-SVM based on quadratic programming. The kernel functions which are frequently used by SVM include linear kernel function, polynomial kernel function, radial basis function, Fourier kernel function, spline kernel function and Sigmoid kernel function [8] [9].

This paper based on the parameter optimization by SVM and AFSA, realizes the intelligent identification of image features. First, it analyzes the SVM classification process, and identifies the important factors which affect SVM classification performance. Then, it selects the image feature information of different objects as the classification criteria, pre-processes the feature information and removes the influence of dimensionality and order of magnitude on classification effect. Finally, after AFSA realizes the parameter optimization of SVM, the test experiment proves that the AFSA-SVM in this paper is effective.

\section{IMAGE FEATURES}

\section{A. Color Feature}

Color feature is a kind of global feature, which describes the surface properties of the scene corresponding to the image or the image area. The color feature describes the surface layer properties of the image area or the whole image corresponding to the scene. The color feature has little dependence on the size and perspective of the image itself, so it has high robustness. Color feature is based on the characteristics of pixels, and any pixel belonging to the image region or the whole image has its own contribution. Because the color is not sensitive to the direction and size of the image or the image area, the color feature can not capture the local features of the object in the image well [11]. Color histogram is a global color feature extraction and matching method. The values in the histogram are statistically derived. It describes the quantitative characteristics of the color in the image. It can reflect the statistical distribution and basic tone of the image color. It can simply describe the global distribution of the color in an image, that is, the proportion of different colors in the whole image Some images that are difficult to be segmented automatically and images that do not need to consider the spatial position of objects. Any color distribution in an image can be represented by its moments. Because the information of color distribution is mainly concentrated in low order moments, the first, second and third moments of color are enough to express the color distribution of images [12].

\section{B. Texture Feature}

Texture feature is a kind of image feature which shows the homogeneity phenomenon in the image. It is also a global feature. This type of feature can reflect the periodic change of the object surface or the organization structure arrangement attribute with slow change. Different from color feature, texture feature is not based on pixel feature, it needs statistical calculation in the region containing multiple pixels. In pattern matching, this regional feature has great advantages and will not fail to match due to local bias [13]. As a statistical feature, texture features often have rotation invariance and good anti noise performance. Texture features not only represent the global characteristics, but also describe the surface characteristics of the image area or the whole image. They are not based on a single pixel, but need to be calculated and counted when the region contains multiple pixels. Texture features depict the repeated local patterns and their arrangement rules, which are often used in image classification and scene recognition. It can only reflect the characteristics of the object surface, can not fully reflect the essential attributes of the object, so only using texture features can not obtain the high-level content of the image. Texture features include nonuniformity of gray distribution, energy, gray average, gradient average, gray mean square error, gradient mean square error, correlation, gray entropy, etc [14].

\section{Shape Features}

The expression of shape features must be based on the segmentation of objects or regions in the image. Two classical algorithms are SIFT and HOG. SIFT is to find the feature points first, while HOG is to segment the image.

(1) SIFT algorithm 
SIFT algorithm to achieve feature matching mainly has three processes, the first is to extract key points, key points are some very prominent will not disappear due to illumination, scale and rotation and other factors, such as corner points, edge points, bright spots in dark areas and dark spots in bright areas, which is to search the image position in all scale space, through the Gaussian differential function to identify potential scale And rotation invariant points of interest. Then, the key points are located and the feature direction is determined. At each candidate position, a fine fitting model is used to determine the position and scale. The selection of key points depends on their stability, and each key point is assigned one or more directions based on the local gradient direction of the image. All operations on image data are transformed relative to the direction, scale and position of key points, so as to provide invariance for these transformations. Finally, by comparing the feature vectors of each key point, the matching pairs of feature points are found, and the corresponding relationship between image objects is established.

The local feature points extracted by SIFT in scale space. SIFT feature points extraction is more convenient and robust to image scaling and other transformations, which has been widely used. The steps of SIFT algorithm are as follows.

(1) The scale space is constructed and the image is scaled down

(2) Search for key points in scale space, which are corners or inflection points

(3) Remove possible noise points, non maximum suppression

(4) Calculate the direction to construct 128 dimensional eigenvectors, calculate the vector of each feature point, each vector is 128 dimensions.

(2) Hog algorithm

Hog (histogram of oriented gradient) is used to detect the feature description of the object. The feature is constructed by calculating the histogram of gradient direction of the local area of the image. Hog feature is especially suitable for human detection in images. Because hog is operated on the local grid unit of the image, it can keep good invariance to geometric and optical deformation of the image. Under the conditions of coarse spatial sampling, fine directional sampling and strong local optical normalization, as long as the pedestrian can generally maintain upright posture, some subtle body movements can be allowed, which can be ignored without affecting the detection results.

Hog feature steps, It is assumed that each region has key characteristics, so it is necessary to operate on each region.

(1) Input image, gamma normalization

(2) Calculate gradient: calculate the gradient of the image in the horizontal direction and vertical direction by difference, and then get the gradient amplitude and direction of each pixel

(3) The whole window is divided into cells with the same size and non overlapping cells (such as $8 \times 8$ pixels), and the gradient size and direction of each cell are calculated. Then the gradient direction of each pixel is divided into 9 bins in $0-180$ (undirected: 0-180, directed: 0-360). The gradient histogram of each cell is counted to form the descriptor of each cell
(4) $2 \times 2$ adjacent cells are divided into $16 \times 16$ pixel blocks, that is block. Slide the sliding window of block size from left to right and from top to bottom to calculate the histogram vector of gradient direction. The hog feature descriptor of a block is obtained by concatenating the feature descriptors of all cells in a block.

(3) Haar features

Haar features can be divided into four categories: linear features, edge features, point features (center features) and diagonal features. They can also be divided into three categories: edge features, linear features (including diagonal features) and point features (central features). By changing the size and location of feature templates, a large number of features can be listed in the image sub window. The feature obtained by extending the feature prototype in the image sub window is called "rectangular feature"; the value of rectangular feature is called "eigenvalue". The rectangle feature can be located at any position of the image, and its size can also be changed arbitrarily. Therefore, the rectangular feature value is a function of the three factors, namely, the type of the rectangle template, the position of the rectangle and the size of the rectangle. Therefore, the change of category, size and position makes the very small detection window contain a lot of rectangular features.

Rectangular features are sensitive to some simple graphic structures, such as edges and line segments, but they can only describe the structures with specific directions (horizontal, vertical, diagonal), so they are relatively rough. For a $24 * 24$ pixel detection window, the number of rectangular features can reach 160000, and the appropriate rectangular features must be selected by a specific algorithm and combined into a strong classifier to detect. The four feature extraction template rectangles of Haar are shown in Fig.1 (the color block matrix includes horizontal, vertical and oblique 45 degrees).

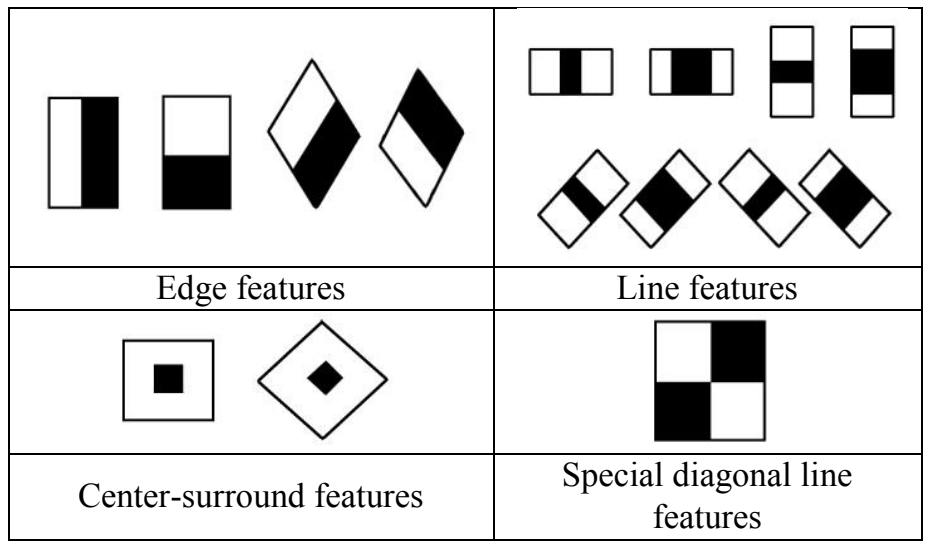

Figure.1 The four feature extraction template rectangles of Haar

\section{(4) LBP features}

LBP (local binary pattern) is an operator used to describe the local texture features of an image. It has the advantages of rotation invariance and gray scale invariance.

The original LBP Operator is defined as: in a $3 * 3$ window, the center pixel of the window is taken as the threshold, and the 
gray value of eight adjacent pixels is compared with it. If the surrounding pixel value is greater than the central pixel value, the position of the pixel is marked as 1 , otherwise it is 0 . In this way, 8 points in the $3 * 3$ neighborhood can be compared to generate 8-bit binary numbers, that is, LBP value of the center pixel of the window is obtained, and this value is used to reflect the texture information of the region.

The biggest drawback of the basic LBP Operator is that it only covers a small area within a fixed radius, which obviously can not meet the needs of different sizes and frequency textures. In order to adapt to the texture features of different scales and meet the requirements of gray scale and rotation invariance, the LBP Operator is improved. The $3 \times 3$ neighborhood is extended to any neighborhood, and the square neighborhood is replaced by the circular neighborhood. The improved LBP Operator allows any number of pixels in the circular neighborhood with radius $\mathrm{R}$. Thus, LBP operators with $\mathrm{P}$ sampling points in a circular region with radius $\mathrm{R}$ are obtained

The extracted LBP Operator can get a LBP code in each pixel. After extracting the original LBP Operator for an image (recording the gray value of each pixel), the original LBP feature is still "a picture" (recording the LBP value of each pixel). In the application of LBP, such as texture classification and face analysis, LBP spectrum is not used as feature vector for classification and recognition, but the statistical histogram of LBP feature spectrum is used for classification and recognition.

The steps of extracting LBP feature vector.

(1) Firstly, the detection window is divided into $16 \times 16$ small cells;

(2) For a pixel in each cell, the gray values of the eight adjacent pixels are compared with it. If the value of the surrounding pixel is greater than that of the center pixel, the position of the pixel is marked as 1 , otherwise it is 0 . In this way, the 8 points in the $3 * 3$ neighborhood can generate 8 -bit binary numbers, that is, the LBP value of the center pixel of the window can be obtained;

(3) Then calculate the histogram of each cell, that is, the frequency of each number (assuming that it is a decimal number LBP value), and then normalize the histogram;

(4) Finally, the statistical histogram of each cell is connected to form a feature vector, which is the LBP texture feature vector of the whole image;

Then SVM or other machine learning algorithms can be used for classification. Several LBP operators are shown in Fig.2.

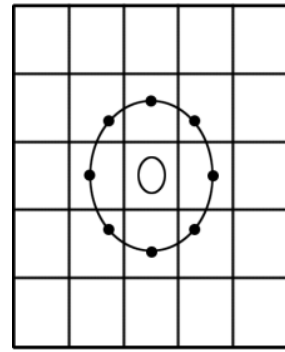

$\operatorname{LBP}_{8}{ }^{1}$

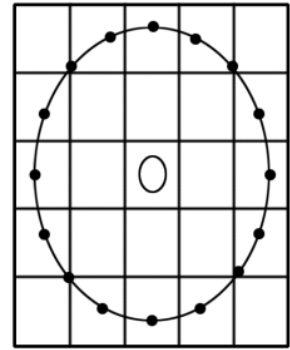

$L B P_{12}{ }^{2}$

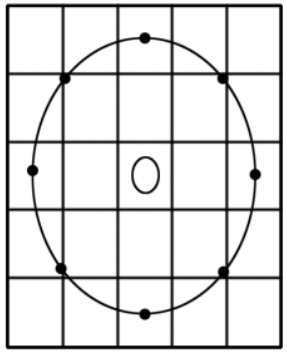

$\mathrm{LBP}_{8}^{2}$
Figure.2 Several LBP operators

\section{PRINCIPLES OF SUPPORT VECTOR MACHINE}

Assume that the training set samples have N-dimensional features and L sample set can be represented as $\left(x_{1}\right), y_{1}, \cdots,\left(x_{l}, y_{l}\right) \in R^{n}$.

Build the hyper-plane as:

$$
f(x)=\varpi \cdot \varphi(x)+b
$$

In this equation, $\varpi$ is the normal vector of the hyper-plane, $\varphi(x)$ is the non-linear mapping function and $b$ is the deviation value [15] [16].

To minimize the structural risk, the optimal classification plane meets the following condition:

$$
y_{i}\left(\varpi \cdot \varphi\left(x_{i}\right)+b\right) \geq 1
$$

Introduce non-negative relaxation factor $\xi_{i}$ to make the classification error within a certain scope. Therefore, the optimization problem has become:

$$
\left\{\begin{array}{l}
\min \frac{1}{2}\|\varpi\|^{2}+c \sum_{i=1}^{n} \xi_{i}, c \geq 0 \\
\text { s.t } \quad y_{i}\left[\left(\varpi \cdot \varphi\left(x_{i}\right)+b\right)\right] \geq 1-\xi_{i}, \quad \xi_{i} \geq 0
\end{array}\right.
$$

where $c$ is the penalty factor, which controls the complexity and generalization of the model [17].

Standard SVM adopts $\varepsilon$-insensitivity function, namely that assume all training data are shown with linear function fitting in accuracy $\varepsilon$,

$$
\left\{\begin{array}{l}
y_{i}-f\left(x_{i}\right) \leq \varepsilon+\xi_{i} \\
f\left(x_{i}\right)-y_{i} \leq \varepsilon+\xi_{i}^{*} \\
\xi_{i}, \xi_{i}^{*} \geq 0
\end{array} \quad i=1,2, \ldots, n\right.
$$

Where $\xi_{i}, \xi_{i}^{*}$ are relaxation factors. When the classification has an error, $\xi$ and $\xi_{i}^{*}$ both are bigger than 0 and they are 0 if there is no error [18]. At that time, this problem has been converted into the minimization problem of the object function:

$$
R\left(\omega, \xi, \xi^{*}\right)=\frac{1}{2} \omega \cdot \omega+C \sum_{i=1}^{n}\left(\xi_{i}+\xi_{i}^{*}\right)
$$

The $1^{\text {st }}$ item in Equation (5) makes the fitting function flatter and thus generalization ability is enhanced. The $2^{\text {nd }}$ item is to reduce the error. The constant $C>0$ means the penalty imposed on the sample which exceeds the error $\varepsilon$. It can be seen by solving Equation (4) and Equation (5) that this is a convex quadratic optimization problem [19]. So Lagrange 
function is introduced to convert the minimization problem into the form of duality:

$$
\left\{\begin{array}{l}
\min \frac{1}{2} \sum_{i=1}^{n} \sum_{j=1}^{n} y_{i} y_{j} a_{i} a_{j} K\left(x_{i}, x_{j}\right)-\sum_{i=1}^{n} a_{i} \\
\text { s.t } \quad \sum_{i=1}^{n} y_{i} a_{i}=0,0 \leq a_{i} \leq c
\end{array}\right.
$$

where

$$
K\left(x_{i}, x_{j}\right)=\left(\varphi\left(x_{i}\right) \cdot \varphi\left(x_{j}\right)\right)
$$

Introduce radial basis function as:

$$
\begin{gathered}
K\left(x_{i}, x_{j}\right)=\exp \left(-g\left\|x_{i}-x_{j}\right\|\right)^{2} \\
K\left(x, x_{i}\right)=\exp \left\{-\frac{\left|x-x_{i}\right|^{2}}{\sigma^{2}}\right\}
\end{gathered}
$$

where $g$ is a kernel function parameter and it decides the scope and width of the input space. Here, the center of every basis function corresponds to a support vector. They as well as the output weights are automatically determined by the algorithm. The inner product function in the form of radial basis resembles human visual system. When different $\mathrm{S}$ parameter values are selected, the corresponding classification plane changes dramatically [20].

The above optimization problem has been converted into:

$$
\left\{\begin{array}{l}
\min \frac{1}{2} \sum_{i=1}^{n} \sum_{j=1}^{n} y_{i} y_{j} a_{i} a_{j} \exp \left(-g\left\|x_{i}-x_{j}\right\|\right)^{2}-\sum_{i=1}^{n} a_{i} \\
\text { s.t } \quad \sum_{i=1}^{n} y_{i} a_{i}=0,0 \leq a_{i} \leq c
\end{array}\right.
$$

Two important parameters in the classification process are penalty factor $c$ and kernel function parameter $g$. To select the effective and optimal $c$ and $g$ makes SVM show excellent prediction performance. If $c$ is too small, under-fitting phenomenon will occur in the model and if $c$ is too big, the model will have over-fitting; if $g$ is too small, the model is not sufficiently accurate and if $g$ is too big, prediction error may occur easily [21] [22].The kernel functions of SVM are shown in Fig 3.

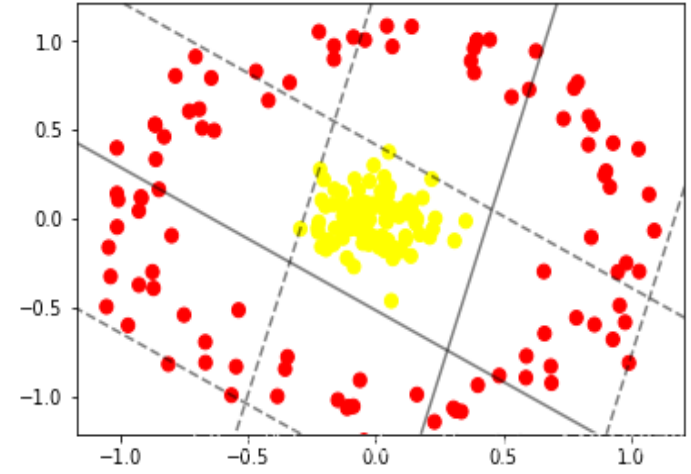

(a) Linear kernel function

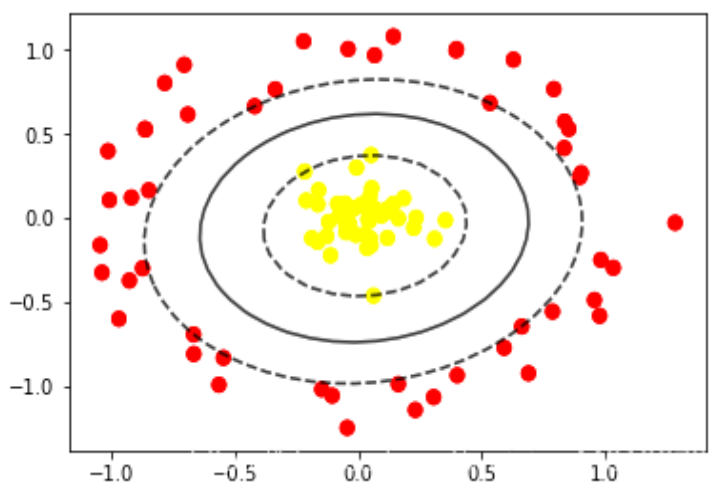

(b) RBF kernel function

Figure.3 The Kernel functions of SVM

\section{ALGORITHM IMPLEMENTATION AND EXPERIMENTAL TEST}

\section{A. Algorithm Process}

Artificial fish swarm algorithm (AFSA) has five basic parameters: visual field of artificial fish, step, group size $n$, trynumber, crowding factor $\delta$.

(1) Visual field: since visual field has a great influence on each behavior of the algorithm, its change has a complex influence on the convergence performance. When the field of vision is small, the foraging behavior and random behavior of artificial fish are more prominent; when the field of vision is large, the rear end behavior and clustering behavior of artificial fish will become more prominent, and the complexity of the corresponding algorithm will also increase. In general, the larger the field of vision, the easier it is for artificial fish to find the global optimal solution and converge.

(2) Step length: for a fixed step length, the convergence speed is accelerated with the increase of the step length. However, if the step length exceeds a certain range, the convergence speed will slow down. If the step length is too large, the oscillation phenomenon will appear, which greatly affects the convergence speed. The random step size method can prevent the oscillation phenomenon to a certain extent, and 
greatly reduce the sensitivity of the parameter, but the fastest convergence rate is the optimal fixed step size. Therefore, for specific optimization problems, we can consider using appropriate fixed step size or variable scale method to improve the convergence rate.

(3) Group size n: the more the number of artificial fish, the stronger the ability to jump out of the local optimal solution, and the faster the convergence speed. Of course, the cost is that the computation of each iteration of the algorithm is also larger. Therefore, in the use process, under the premise of stable convergence, the number of graphs should be reduced as much as possible.

(4) Trynumber: the more the number of attempts, the stronger the foraging ability of artificial fish and the higher the convergence efficiency. When the local extremum is prominent, it should be appropriately reduced to increase the probability of artificial fish random walk and overcome the local optimal solution.

(5) Crowding factor $\delta$ : take the maximum value as an example (the minimum value is just opposite to the maximum value). The larger the $\delta$ is, the smaller the allowable crowding degree is, and the stronger the artificial fish's ability to get rid of the local optimal solution is. However, the convergence speed will slow down. This is mainly because the artificial fish will walk away randomly due to avoiding overcrowding or be rejected by other artificial fish when approaching the optimal solution. It can't approach the extremum point accurately by using the method. It can be seen that although the introduction of $\delta$ can avoid the artificial fish from overcrowding and falling into the local optimal solution, on the other hand, this parameter will make the artificial fish near the extreme point repel each other and it is difficult to approach the extreme point accurately. Therefore, for some specific problems where the local extremum is not very serious, the crowding factor can be ignored, so as to simplify the algorithm, accelerate the convergence speed and improve the accuracy of the results.

Randomly select 60 groups of every type of different microbial samples as the training set and 20 groups as prediction set. First of all, adopt data pre-processing method and remove the impact of different dimensions and magnitudes in 20-dimensional image features on the classification performance. Then select AFSA to optimize the selection of important parameters which affect SVM classification performance. The initial population is 30 , the dimension is 20 , the step length is 0.5 , the visible range is $2, \delta$ is 0.6 and the trial of foraging behavior is 10 . The basic behaviors of artificial fish are as follows.

(1) Foraging behavior

The current status of artificial fish $i$ is $X_{i}$ and randomly select a state $X_{j}$ in its perception range.

$$
X_{j}=X_{i}+\text { Visual } \cdot \text { Rand() }
$$

where $\operatorname{Rand}()$ is a random figure between 0 and 1 . In the maximization problems, if $Y_{i}<Y_{j}$, move one step towards this direction.

$$
X_{i}^{t+1}=X_{i}^{t}+\frac{X_{j}-X_{i}^{t}}{\left\|X_{j}-X_{i}^{t}\right\|} \cdot \text { Step } \cdot \operatorname{Rand}()
$$

Otherwise, randomly select the state $X_{j}$ again, and determine whether the condition to move forward is met. If not, randomly move one step forward.

$$
X_{i}^{t+1}=X_{i}^{t}+\text { Visual } \cdot \operatorname{Rand}()
$$

\section{(2) Swarm behavior}

The current state of the artificial fish is $X_{i}$ and explore the number of fish $n_{f}$ which meets $d_{i j}<$ Visual and the central position $X_{o}$ in the current neighborhood. If $Y_{c} / n_{f}>\delta Y_{i}$, it indicates that there is much food in the center and it is not crowded. Then move one step towards the center.

$$
X_{i}^{t+1}=X_{i}^{t}+\frac{X_{c}-X_{i}^{t}}{\left\|X_{c}-X_{i}^{t}\right\|} \cdot \text { Step } \cdot \operatorname{Rand}()
$$

Otherwise, implement the foraging behavior.

(3) Following behavior

The current state of artificial fish $i$ is $X_{i}$. Explore the fish $X_{j}$ with the maximum $Y_{j}$ among those which meet $d_{i j}<$ Visual in the current neighborhood. If $Y_{j} / n_{f}>\delta Y_{i}$, it indicates that the state of $X_{j}$ has higher food concentration and it is not very crowded. Then move one step towards the direction of $X_{j}$.

$$
X_{i}^{t+1}=X_{i}^{t}+\frac{X_{j}-X_{i}^{t}}{\left\|X_{j}-X_{i}^{t}\right\|} \cdot \text { Step } \cdot \operatorname{Rand}()
$$

Otherwise, foraging behavior is implemented.

The above three kinds of behaviors will convert into each other in different conditions. The fish selects the current optimal behavior for implementation through the evaluation of the behavior to reach the position with higher food concentration.

\section{B. Experiment and Analysis}

The test images come from the images of microorganisms in the field of biology. Altogether 20 dimensions are extracted from the color features and texture features of different images. RBF is selected as the classification kernel function of SVM. Fig. 4 and Fig. 5 are the parameter optimization process and result of AFSA-SVM. It can be seen that when the training set is 4 times crossover and the accuracy is $100 \%$, the optimal 
parameters $c$ and $g$ are obtained and they are 15.1832 and 0.068754 respectively.

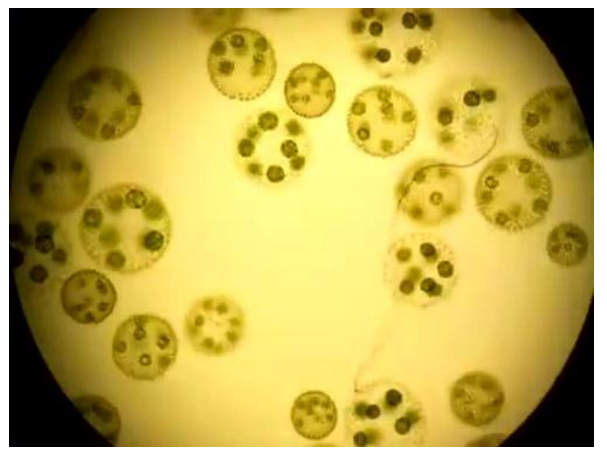

(a)Original image

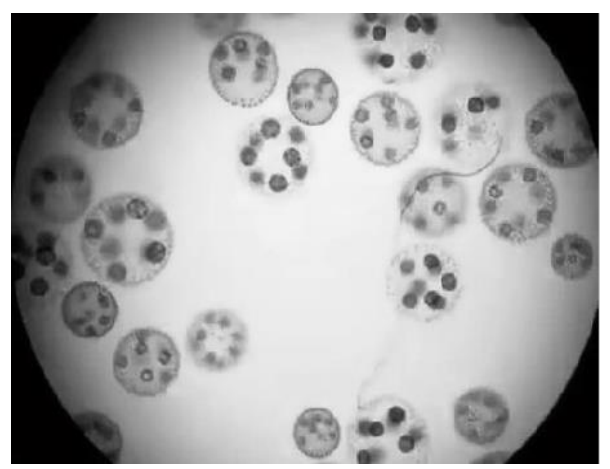

(b)Grayscale image

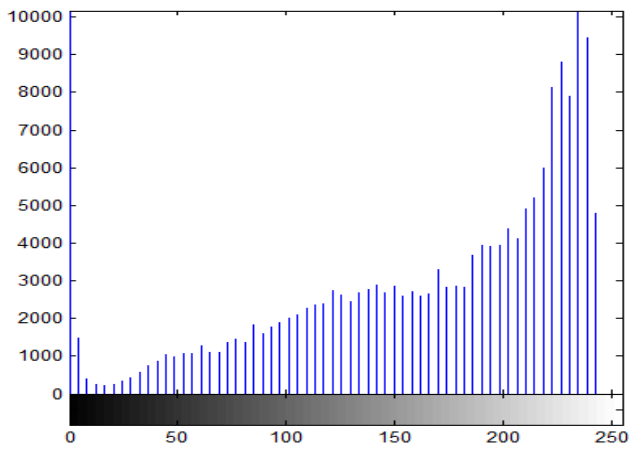

(c)Histogram

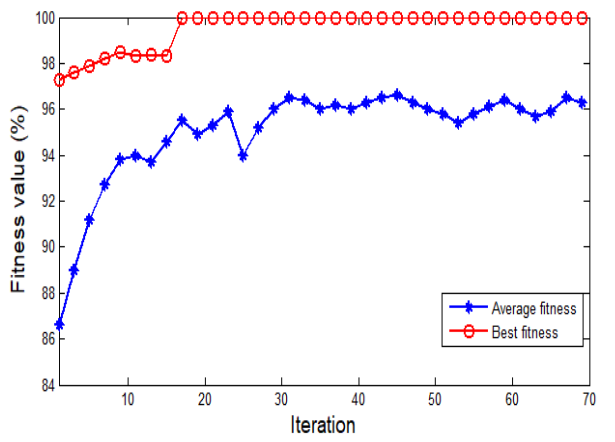

(d)Parameter optimization process of AFSA-SVM

Figure.4 Microorganism 1 in water

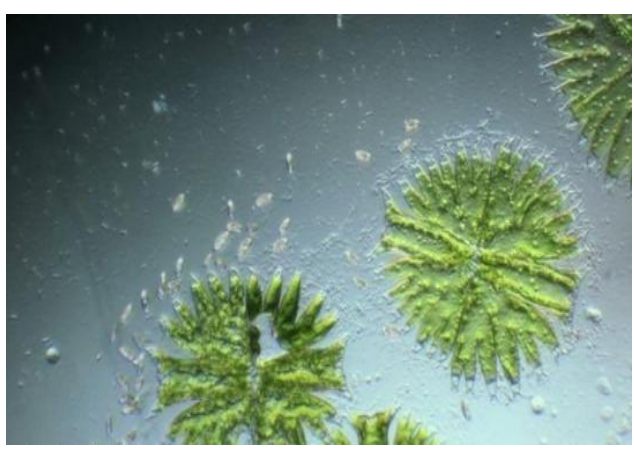

(a)Original image

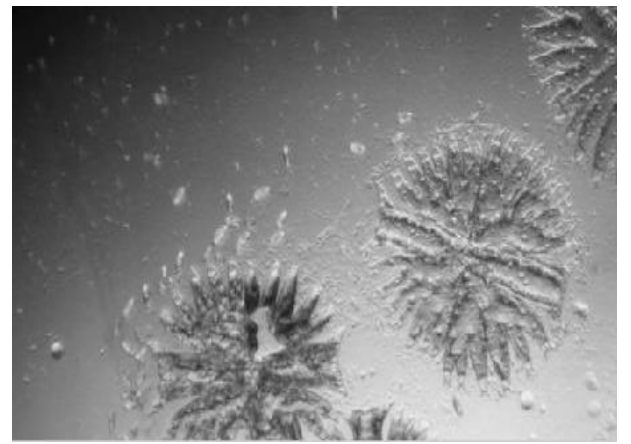

(b)Grayscale image

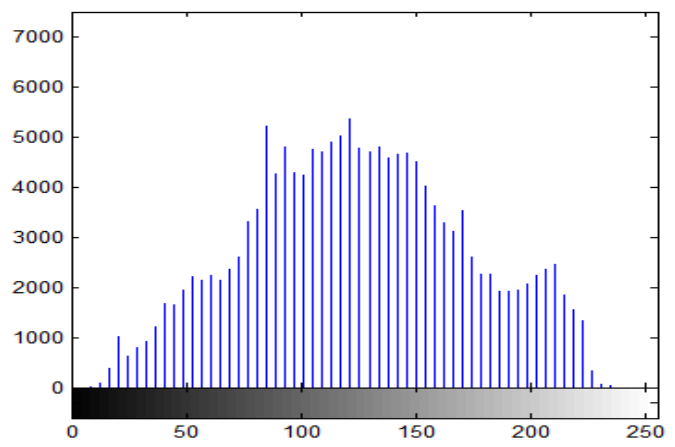

(c)Histogram

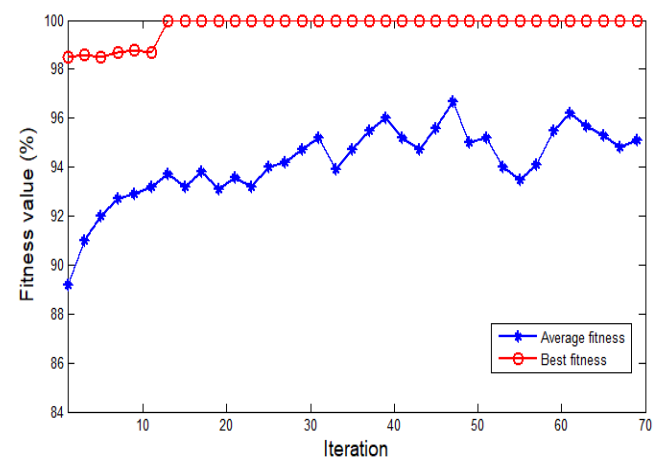

(d)AFSA-SVM Parameter optimization process of AFSA-SVM

Figure.5 Microorganism 2 in water

As shown in the above experimental results, AFSA-SVM has better classification accuracy for the feature data of 
different microbes. Whether the sample data is high- or low-dimensional, and whether there is a great or a small data volume, RBF shows perfect classification performance. In other words, the small error obtained from definite trainings samples can make sure that the independent testing set still preserves a small error. With excellent generalization ability, the problems to be solved by AFSA-SVM correspond to a convex optimization problem; so the local optimal solution must be the global optimal solution. RBF converts non-linear problems into linear problems to find the solutions. The maximization of classification interval makes SVM has strong robustness. SVM is selected as a pattern classifier for intelligent recognition of image characteristic data. After theoretical analysis, it can be found that both penalty factor and kernel function parameter are important when it comes to the influence on SVM classification performance.

The decision boundary of SVM is the maximum margin hyperplane to solve the learning samples. The advantage of SVM is that the generalization ability of SVM is strong, which can prove the generalization error of SVM. SVM solves the nonlinear classification problem by using the idea of spatial mapping and kernel function, and avoids the disaster of dimension. SVM has good robustness, and its final model "discards" a large number of redundant samples, only related to support vector, the latter only occupies a small proportion. For the case that the edges of two classification data overlap, the classification effect of SVM is not good enough. To solve this problem, SVM uses relaxation factor to "soften" the boundary. In order to achieve better fitting results, it allows some points to be within the boundary. The "hardness" of the boundary line can be controlled by a super parameter, usually C. If the relaxation variable $\mathrm{C}$ is large, the boundary will be "hard", and the data points can not "survive" within the boundary; if $\mathrm{C}$ is small, the boundary line is "soft", and some data points can cross the boundary line.

\section{CONCLUSION AND FUTURE WORK}

Due to its high flexibility and accuracy, microbial detection technology has been widely applied in medicine and food safety detection. Classifier has played a critical role as the terminal of pattern recognition process, which requires the algorithm to have strong non-linear mapping capability, data parallel processing capacity and generalization ability. With the development of artificial intelligence, many intelligent algorithms have come into being. SVM has been extensively used as the mainstream classifier in the current artificial intelligence field. AFSA is a concrete application of swarm intelligence. It needs no knowledge of particular information of the problems; instead, it only compares the problems and it has a fast convergence speed. This paper, based on using AFSA in the parameter optimization of SVM, has achieved the intelligent detection of microbial image features. The test experiment has proven that AFSA-SVM has better microbial image feature recognition.
This paper combines SVM and artificial fish swarm algorithm (AFSA) for parameter optimization, builds AFSA-SVM classification model to achieve the intelligent identification of image features, and provides reliable technological means to accelerate sensing technology. The parameter selection of support vector machine often affects the performance of classifier. The parameter selection method in this paper is obtained by intelligent optimization algorithm AFSA, which effectively improves the generalization ability of support vector machine. The hybrid AFSA-SVM algorithm has faster convergence speed and more accurate accuracy. The future research work is as follows.

(1) The next step is to optimize the objective function or design other fuzzy membership functions to further improve the robustness of AFSA-SVM.

(2) In the case of seriously unbalanced data samples, SVM classification results tend to be biased to the one with large amount of data. How to effectively avoid the impact of unbalanced sample information on SVM is one of the important contents of future research.

(3) The selection range of SVM parameters is not clearly defined. If the selection range is too large or too small, the phenomenon of "over fitting" and "under fitting" may appear in the learning machine. How to effectively avoid this situation and give different parameter selection ranges for different problems are also one of the key contents of future research.

\section{REFERENCE}

[1] Yin Cong, Luan Qiuping, Feng nianlun, "Study on Microscopic Image Analysis and Recognition Technology of Pathological Cells", Biomedical engineering research, 2009, vol. 28, no. 1, pp.35-38.

[2] Suykens J A K, Vandewalle J., "Least Squares Support VectorMachine Classifiers", Neural Processing Letters, 2012, vol. 9, no. 3, pp.293-300.

[3] Imran Razzak, Khurram Zafar, Muhammad Imran, Guandong Xu. (2020) "Randomized Nonlinear One-Class Support Vector Machines with Bounded Loss Function to Detect of Outliers for Large Scale IoT Data", Future Generation Computer Systems, 112, pp.715-723.

[4] Julio López, Sebastián Maldonado, Miguel Carrasco, "Robust Nonparallel Support Vector Machines Via Second-Order Cone Programming", Neurocomputing, 2019, vol. 364, no. 28, pp.227-238.

[5] Lukas L, Suykens J, Vandewalle J., "Least Squares Support Vector Machines Classifiers : A Multi Two-Spiral Benchmark Problem", Proc of the Indonesian Student Scientific Meeting, 2011, vol. 3, no.11, pp.289-292.

[6] B. Richhariya, M. Tanveer, "EEG Signal Classification Using Universum Support Vector Machine", Expert Systems with Applications, 2018, vol.106, no.15, pp.169-182.

[7] Deepak Kumar, Manoj Thakur, "All-In-One Multicategory Least Squares Nonparallel Hyperplanes Support Vector Machine", Pattern Recognition Letters, 2018, vol.105, no.1, pp.165-174.

[8] Márcio Dias de Lima, Nattane Luiza Costa, Rommel Barbosa, "Improvements on Least Squares Twin Multi-Class Classification Support Vector Machine", Neurocomputing, 2018, vol.313, no.3, pp.196-205.

[9] Boyuan Ma, Xiaojuan Ban, Ya Su, Chuni Liu, Di Wu, "Fast-Finecut: Grain Boundary Detection In Microscopic Images Considering 3D Information”, Micron, 2019, vol.116, no.1, pp. 5-14.

[10] Gerrit G. Tamminga, Astrid H. Paulitsch-Fuchs, Gijsbert J. Jansen, Gert-Jan W. Euverink, "Development and Validation of an Alternative Parameter for Quantification of Signals Emitted By Fluorescently Labelled Bacteria in Microscopic Images", Journal of Microbiological Methods, 2019, vol.166, no.11, article.105717. 
[11] A. E. Parker, J. A. Christen, L. Lorenz, H. Smith, "Optimal Surface Estimation and Thresholding of Confocal Microscope Images of Biofilms Using Beer's Law", Journal of Microbiological Methods, 2020, vol.174, no.7, article.105943.

[12] Yane Duan, Daoliang Li, Lars Helge Stien, Zetian Fu, Yongping Gao. "Automatic Segmentation Method For Live Fish Eggs Microscopic Image Analysis", Aquacultural Engineering, 2019, vol.85, no.5, pp.49-55.

[13] Du, Shenglian; Wang, Yuemei, "Concrete Pore Structure under Vibration Based on Microscope Image Analysis", Acta Microscopica , 2019, vol.28, no.3, pp.509-519.

[14] Wang, Xijuan, "Real-time Respiratory Motion Image Processing Prediction", Investigacion Clinica, 2019, vol.60, no.3, pp.619-630.

[15] Kumar Manoj Prabhakaran, Rajagopal Manoj Kumar, "Detecting Facial Emotions Using Normalized Minimal Feature Vectors and Semi-Supervised Twin Support Vector Machines Classifier", Applied Intelligence, 2019, vol.49, no.12, pp.4150-4174.

[16] Furukawa, Osamu, "A Study on Thermal Detection Based on Support Vector Machine Using Dynamic Time Warping and Application to Optical Fiber Sensor", IEEE Sensors Journal, 2021, vol.21, no.5, pp. 6325-6334.

[17] Zidane Flora, Lanteri Jerome, Brochier Laurent, etc, "Damaged Apple Sorting with mmWave Imaging and Nonlinear Support Vector Machine", IEEE Transactions on Antennas and Propagation, 2020, vol. 68, no.12, pp. 8062-8071.

[18] Yang Qifan, Zhang Huijuan, Xia Jun, etc, "Evaluation of Magnetic Resonance Image Segmentation in Brain Low-Grade Gliomas Using Support Vector Machine and Convolutional Neural Network",
Quantitative Imaging in Medicine and Surgery, 2021, vol.11, no.1, pp. 300-316.

[19] Zhao Meng, Liu Jingjing, Cai Wanye, etc, "Support Vector Machine Based Classification of Smokers and Nonsmokers Using Diffusion Tensor Imaging", Brain Imaging and Behavior, 2020, vol.14, no.6, pp. 2242-2250.

[20] Xu Shuo, An Xin, “(MLS)-S-2-SVM: Multi-Label Least-Squares Support Vector Machine Classifiers", Electronic Library, 2019, vol.37, no.6, pp.1040-1058.

[21] Zhang, Qisong; Li, Yingqiu; Yang, Xiaoguang, "Digital Planting and Management System for Crops", Revista De La Facultad De Agronomia De La Universidad Del Zulia, vol. 36, no.4, pp.1145-1155.

[22] Niam Abdulmunim Al-Thanoon, Omar Saber Qasim, Zakariya Yahya Algamal, "Tuning Parameter Estimation in SCAD-Support Vector Machine Using Firefly Algorithm With Application In Gene Selection and Cancer Classification", Computers in Biology and Medicine, 2018, vol.103, no.1, pp.262-268.

\section{Creative Commons Attribution License 4.0 (Attribution 4.0 International, CC BY 4.0)}

This article is published under the terms of the Creative Commons Attribution License 4.0 https://creativecommons.org/licenses/by/4.0/deed.en_US 